\section{This paper is published as part of a PCCP themed issue on electronic energy transfer}

\author{
Guest Editor: Anthony Harriman
}

Editorial

Electronic energy transfer

Anthony Harriman, Phys. Chem. Chem. Phys., 2010

DOI: $10.1039 / \mathrm{cocp} 90032 \mathrm{j}$

Perspectives

Quantum coherence and its interplay with protein environments in photosynthetic electronic energy transfer

Akihito Ishizaki, Tessa R. Calhoun, Gabriela S. Schlau-

Cohen and Graham R. Fleming, Phys. Chem. Chem.

Phys., 2010

DOI: $10.1039 / \mathrm{c003389h}$

Excitation energy transfer in donor-bridge-acceptor systems

Bo Albinsson and Jerker Mårtensson, Phys. Chem.

Chem. Phys., 2010

DOI: $10.1039 / \mathrm{c003805a}$

Physical origins and models of energy transfer in photosynthetic light-harvesting

Vladimir I. Novoderezhkin and Rienk van

Grondelle, Phys. Chem. Chem. Phys., 2010

DOI: $\underline{10.1039 / c 003025 b}$

\section{Communication}

Formation and energy transfer property of a subphthalocyanine-porphyrin complex held by hostquest interactions

Hu Xu, Eugeny A. Ermilov, Beate Röder and Dennis K. P. Ng, Phys. Chem. Chem. Phys., 2010

DOI: $10.1039 / \mathrm{c} 004373 \mathrm{~g}$

Papers

Charge transfer in hybrid organic-inorganic $\mathrm{PbS}$ nanocrystal systems

Muhammad N. Nordin, Konstantinos N. Bourdakos and

Richard J. Curry, Phys. Chem. Chem. Phys., 2010

DOI: $10.1039 / \mathrm{c} 003179 \mathrm{~h}$

Superexchange-mediated electronic energy transfer in a model dyad

Carles Curutchet, Florian A. Feist, Bernard Van

Averbeke, Benedetta Mennucci, Josemon Jacob, Klaus

Müllen, Thomas Basché and David Beljonne, Phys.

Chem. Chem. Phys., 2010

DOI: $10.1039 / \mathrm{c003496g}$

\section{PCCP}

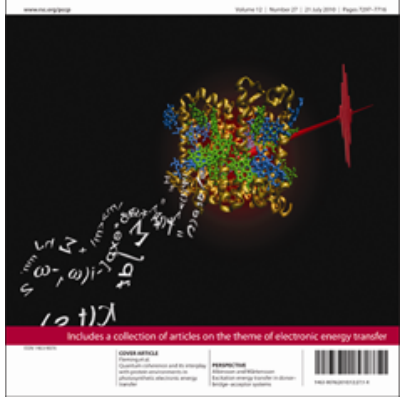

Switching off FRET by analyte-induced decomposition of squaraine energy acceptor: A concept to transform 'turn off' chemodosimeter into ratiometric sensors

Haibo Yu, Meiyan Fu and Yi Xiao, Phys. Chem. Chem. Phys., 2010

DOI: $10.1039 / \mathrm{c} 001504 \mathrm{k}$

Hybrid complexes: Pt(II)-terpyridine linked to various acetylide-bodipy subunits

Francesco Nastasi, Fausto Puntoriero, Sebastiano Campagna, Jean-Hubert Olivier and Raymond Ziessel, Phys. Chem. Chem. Phys., 2010

DOI: $10.1039 / \mathrm{c} 003789 \mathrm{c}$

Conformational dependence of the electronic coupling for singlet excitation energy transfer in DNA. An INDO/S study

Alexander A. Voityuk, Phys. Chem. Chem. Phys., 2010

DOI: $10.1039 / \mathrm{c003131 \textrm {C }}$

On the conveyance of angular momentum in electronic energy transfer

David L. Andrews, Phys. Chem. Chem. Phys., 2010

DOI: $10.1039 / \mathrm{c} 002313 \mathrm{~m}$

Isotopic effect and temperature dependent intramolecular excitation energy transfer in a model donor-acceptor dyad Jaykrishna Singh and Eric R. Bittner, Phys. Chem. Chem. Phys., 2010

DOI: $10.1039 / \mathrm{c} 003113 \mathrm{e}$

Photophysics of conjugated polymers: interplay between Förster energy migration and defect concentration in shaping a photochemical funnel in PPV

Sangeeta Saini and Biman Bagchi, Phys. Chem.

Chem. Phys., 2010

DOI: $10.1039 / \mathrm{c} 003217 \mathrm{~d}$

Electronic energy harvesting multi BODIPY-zinc porphyrin dyads accommodating fullerene as photosynthetic composite of antenna-reaction center

E. Maligaspe, T. Kumpulainen, N. K. Subbaiyan, M. E. Zandler, H. Lemmetyinen, N. V. Tkachenko and F. D' Souza, Phys. Chem. Chem. Phys., 2010 DOI: $10.1039 / \mathrm{c002757}$ 


\title{
On the conveyance of angular momentum in electronic energy transfer
}

\author{
David L. Andrews* \\ Received 2nd February 2010, Accepted 24th May 2010 \\ First published as an Advance Article on the web 10th June 2010 \\ DOI: $10.1039 / \mathbf{c 0 0 2 3 1 3 m}$
}

\begin{abstract}
When electronic excitation transfer occurs, it is of considerable interest to establish whether angular momentum can also be conveyed in the process. The question is prompted by a consideration that when the participating chromophores are atoms, ions, or molecular systems having high local symmetry, the electronic excited states that are involved are generally characterized not only by energy, but by angular momentum properties. Moreover, it is known that electron spin can be communicated between quantum dot exciton states. Resolving the general issue entails an electrodynamic representation exploiting irreducible tensor methods, the analysis being illustrated by application to energy transfer associated with a variety of multipolar transitions. The results exhibit novel connections between an angular momentum content of the electromagnetic coupling and a strongly varying distance dependence. It is concluded that the communication of angular momentum does not in general map unambiguously between a donor and energy acceptor.
\end{abstract}

\section{Introduction}

In materials comprising chromophores displaying optically well characterized and distinct absorption and fluorescence, electronic energy acquired by the absorption of light commonly migrates between closely neighbouring chromophores. Beyond the region of significant orbital overlap, the primary means for such a relocation of excitation energy - a process occurring well before complete thermal degradationis a mechanism often referred to as resonance energy transfer. ${ }^{1-6}$ Usage of the term 'resonance' here establishes a connection with other physical systems in which energy migrates from one component to another; the term is no longer used in an earlier sense $^{7}$ that signified a lack of engagement with molecular vibrations. Commonly, resonance energy transfer (RET) is associated with the electric dipole-allowed Förster mechanism, though it is widely acknowledged that especially complex, low-symmetry molecular systems can necessitate more sophisticated theories-see for example recent work by Hsu et al., ${ }^{8}$ and May. ${ }^{9}$

It is no surprise that energy is conserved in the transfer of excitation between chromophores, even if that conservation accommodates some intramolecular dissipation at one or other location. Until recently, however, little attention has been given to the apparently analogous possibility of non-contact angular momentum transfer. A comprehensive analysis, even for lone atoms, is complicated by a need to consider not only electric dipole-allowed transitions, but also those that occur by higher order forms of multipolar coupling. Nonetheless, simple atomic systems are subject to well-known selection rules for electronic transitions, ${ }^{10}$ their discrete energy and angular momentum states reflecting detailed properties that emerge under conditions of local isotropy. This might

School of Chemistry, University of East Anglia, Norwich NR4 7TJ, United Kingdom lead to a suspicion that, in the course of resonance energy transfer, angular momentum could be conveyed, quantum for quantum, between donor and acceptor-the corollary being that the multipolar form of donor decay would necessitate an excitation of the same multipolar form in the acceptor. The possible validity of such an assumption demands resolution.

For molecules, or atoms placed in anisotropic environments, an additional tier of complexity is encountered. In systems where full rotational symmetry is compromised, not every electronic state can be uniquely characterized in terms of angular momentum, nor can each electronic transition be unambiguously associated with a specific angular momentum change; a given transition may be allowed by more than one form of multipolar coupling. Under such circumstances it might be anticipated that no correlation would exist between the multipolar characters of the donor decay and acceptor excitation transitions. Again, it is important to ascertain the truth of such a supposition. Generally, one can pose the question: With what degree of fidelity can the electrodynamic coupling responsible for resonance energy transfer also convey angular momentum information between a given donor and acceptor? The analysis that follows aims to address the key issues. Before proceeding, however, it is worth attending to some other features of contextual relevance.

Certain developments that first came to light twenty years ago impinge significantly on the current issues of angular momentum conveyance. First, it became known from a number of quantum electrodynamical studies that the form of electrodynamic coupling involved in resonance energy transfer is identical to that which radiatively conveys fluorescence to a detector. The key difference is that the latter is a phenomenon generally observed over wave-zone distancesi.e. distances significantly larger than the corresponding optical wavelength - whereas resonance energy transfer operates primarily over near-field, i.e. sub-wavelength distances. What are commonly called 'radiative' and 'radiationless' energy 


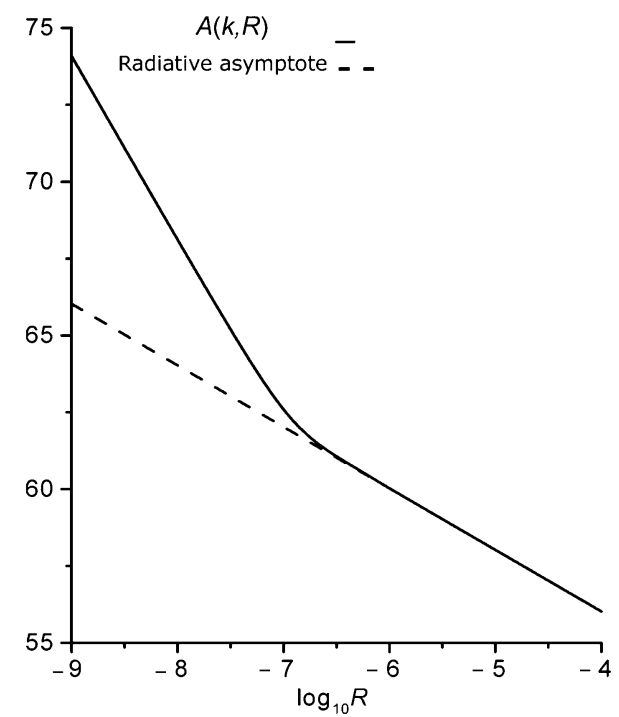

Fig. 1 Log-log plot of the efficiency of electronic energy transfer over distances $R$ (in metres), for a transfer wavelength of $700 \mathrm{~nm}$, vertical scale arbitrary. In the near-field region the gradient is -6 , indicating radiationless transfer; in the long-range asymptote the slope of -2 signifies 'radiative' transfer.

transfer are simply asymptotes of a unified electrodynamic mechanism. ${ }^{11-17}$ In the case of electric dipole-allowed transitions the two distance regimes are characterized by an inverse-square dependence on distance in the wave-zone, inverse sixth power in the near-field. Over intermediate distances additional terms arise, giving rise to an overall distance dependence as exhibited in Fig. 1. In the present context, the significance is a perspective it affords on the fundamental character of energy transfer; the mechanism entails an electrodynamic form of coupling that exhibits increasingly prominent retardation features as distance is increased. This is a fact that will prove important for comprehending the results that emerge.

Over much the same period, parallel advances in quantum optics have led to a dawning appreciation that electromagnetism can convey not only spin angular momentumthrough the familiar connection with circular polarization states - but also what has become known as an orbital angular momentum of light, generally deriving from a helical structure in a propagating wavefront. The terms 'spin' and 'orbital' used in this particular connection have no established connection with the usage of those terms in electronic state designations, but the terminology has become ingrained. ${ }^{18-20}$ Insofar as analogies exist, it is notable that the orbital angular momentum of light is quantized in a sequence of integer values - although there is no upper bound. The capacity to experimentally exploit such features has already evolved into a subject in its own right, and it is a topical area that is generating a rapidly burgeoning range of applications. ${ }^{21,22}$ However, although much interest has focused on optically engineered vortex beams, the capacity for the propagation of orbital angular momentum has much wider scope. ${ }^{19}$ For the present analysis these developments serve as a reminder that electromagnetic fields can convey angular momentum
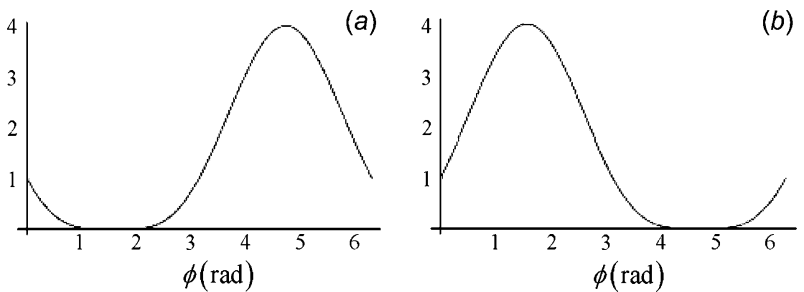

Fig. 2 Quantum dot energy transfer. Variation of (a) spin antiparallel and (b) spin parallel transfer functions as a function of relative orientations. Adapted from Scholes et al. ${ }^{35}$

information that is not restricted to one bit per photon ${ }^{23-25}$ - and hence not just one bit per energy-releasing decay transition.

One further development of contextual relevance is the growing interest in the transfer of electronic excitation between quantum dots, an effect that shows some promise for eventual implementation in quantum computation. Technical developments in this area generally seek to exploit the discrete, size-tunable, and highly intense character of quantum dot exciton transitions, as well as their ultrafast response to optical excitation. More significantly still, the use of circularly polarized excitation can populate specific exciton spin states, enabling the coding of additional information. ${ }^{26-33}$ It has been shown by quantum electrodynamics (QED) analysis that the spin state of an exciton can be transmitted through RET between quantum dots; ${ }^{34,35}$ the plots in Fig. 2 illustrate the effect of rotating one quantum dot relative to another. When the transition moments are parallel, the exciton spin orientation is faithfully communicated from one quantum dot to another; when the moments are antiparallel, excitation transfer causes the spin to flip. Energy migration down a column of quantum dots oriented in a common direction therefore proceeds with a full retention of spin orientation, signifying conservation of angular momentum. The establishment of this result again highlights the need to secure an equally thorough understanding of what wider, more general principles might operate in the interchromophore communication of angular momentum.

\section{Electrodynamic foundations}

The nature of the issues that arise in the conveyance of angular momentum in electronic energy transfer, which requires full consideration of not just electric dipole but also higher order multipole coupling, ${ }^{36,37}$ invites a thorough quantum electrodynamical analysis - a consideration that is reinforced by recognizing the theoretical provenance of the context detailed above. QED treats matter and light on the same fully quantized basis. Amongst its singular features, this formulation of theory has a proven explanatory role, providing physical insights and revealing the fundamental mechanisms for commonplace as well as more exotic phenomena. For example it successfully accounts for single-molecule fluorescence, a process where semiclassical theory surprisingly fails. $^{38-42}$ The reason is plain; for an isolated atom or molecule, any electronically excited eigenstate of the corresponding atomic or molecular Hamiltonian operator is necessarily a stationary state - whereas in QED the additional 
presence of a vacuum radiation field perturbs the system and can induce an electronic decay transition. It has been demonstrated that even the representation of a dipole (indeed any multipole) transition is ultimately legitimate only in a quantum electrodynamical basis. ${ }^{43}$ QED methods are singularly well suited for the analysis of nanoscale photonic interactions, ${ }^{44}$ and they have recently brought to light the possible significance of a change in intermolecular force that accompanies energy transfer-resulting from the fact that pair potentials depend on the electronic state of each molecular component. $^{45,46}$ The same methods have revealed the possibility of optically controlling energy transfer by a non-resonant laser beam, operating through an optically nonlinear mechanism. In systems where electronic energy transfer is designedly precluded by geometry or selection rules, the process can thus effect the function of an optical transistor, acceptor excitation being activated by the laser signal. ${ }^{47,48}$

The following analysis aims to elicit angular momentum and symmetry-related features in electronic energy transfer, to be identified from a suitable development of the founding equations that govern the process of energy transfer. To address resonance energy transfer from $A$ to $B$, the initial and final states of the system (comprising $A, B$ and the radiation field) are written as $\mid \varphi_{\left.A^{*} ; \varphi_{B} ; 0\right\rangle}$ and $\left|\varphi_{A} ; \varphi_{B^{*}} ; 0\right\rangle$, respectively, and the energy transfer rate constant is given by the Fermi rule;

$$
k_{\mathrm{RET}}=\frac{2 \pi}{\hbar}\left|M_{f i}\right|^{2} \rho,
$$

where $M_{f i}$ is the quantum amplitude connecting the initial and final states of the entire system, and $\rho$ a density of states. Although it is not to be pursued here, a development of the density of states in terms of a vibrational structure in the energy levels leads to the familiar dependence of the transfer rate on the spectral overlap between the donor fluorescence and acceptor absorption. ${ }^{13}$ The evaluation of eqn (1) requires a determination of the quantum amplitude. For this purpose time-dependent perturbation theory is deployed, beginning with the exact multipolar (Power-Zienau-Woolley, PZW) Hamiltonian: ${ }^{49-52}$

$$
H=H_{A}+H_{B}+H_{\mathrm{int}}(A)+H_{\mathrm{int}}(B)+H_{\mathrm{rad}} .
$$

The first two terms in (2) denote the unperturbed Hamiltonian operators for the two chromophores, and the $H_{\text {int }}$ operators represent their corresponding interactions with the radiation field in an arbitrary state. The final term, $H_{\mathrm{rad}}$, is the radiation Hamiltonian. Since both $H_{\text {int }}$ terms and $H_{\text {rad }}$ are operators, they are part of the sum whether or not photons are present. The absence of any term in eqn (2) directly linking $A$ with $B$ signifies that every form of electrodynamic interaction between the two must be mediated by interactions with the radiation field.

It is expedient to focus on electric multipole transitions, for reasons that will emerge. From the detailed form of the PZW quantum electrodynamical Hamiltonian, the individual interaction terms in (2) can be determined from;

$$
H_{\mathrm{int}}(\xi)=-\varepsilon_{0}^{-1} \int \boldsymbol{p}^{\perp}(\xi, \boldsymbol{r}) \cdot \boldsymbol{d}^{\perp}(\mathbf{r}) \mathrm{d}^{3} \boldsymbol{r}
$$

in which the radiation operator $\boldsymbol{d}^{\perp}(\mathbf{r})$ represents the transverse electric displacement field, and the matter operator $\boldsymbol{p}^{\perp}(\xi, \boldsymbol{r})$ is the transverse electric polarization. The latter is concisely expressible as follows, defined in terms of a summation that is taken over all of the composite charges $e_{\alpha}$, at positions $\boldsymbol{q}_{\alpha}$, within the donor/acceptor $\xi$ located at $\boldsymbol{R}_{\xi}: 52$

$$
\boldsymbol{p}^{\perp}(\xi, \boldsymbol{r})=\sum_{\alpha(\xi)} e_{\alpha}\left(\boldsymbol{q}_{\alpha}-\boldsymbol{R}_{\xi}\right) \int_{0}^{1} \delta^{\perp}\left(\boldsymbol{r}-\boldsymbol{R}_{\xi}-\lambda\left(\boldsymbol{q}_{\alpha}-\boldsymbol{R}_{\xi}\right)\right) \mathrm{d} \lambda .
$$

where $\delta^{\perp}$ is the transverse Dirac delta function. The expansion of eqn (4) in powers of $\lambda$ delivers the electric multipolar series in its entirety, and $H_{\text {int }}(\xi)$ is expressible as;

$$
\begin{aligned}
H_{\mathrm{int}}(\xi)= & -\varepsilon_{0}^{-1} \boldsymbol{\mu}(\xi) \cdot \boldsymbol{d}^{\perp}\left(\boldsymbol{R}_{\xi}\right)--\varepsilon_{0}^{-1} Q(\xi): \nabla \boldsymbol{d}^{\perp}\left(\boldsymbol{R}_{\xi}\right) \\
& -\varepsilon_{0}^{-1} \Omega(\xi) \vdots \nabla \nabla \boldsymbol{d}^{\perp}\left(\boldsymbol{R}_{\xi}\right)-\ldots
\end{aligned}
$$

Using the Einstein summation convention for repeated Cartesian indices, the leading, electric dipole term can be written as $-\varepsilon_{0}^{-1} \boldsymbol{\mu}(\xi) \cdot \boldsymbol{d}^{\perp}\left(\boldsymbol{R}_{\xi}\right)$, the second, electric quadrupole term as $-\varepsilon_{0}^{-1} Q_{i j}(\xi) \nabla_{j} \boldsymbol{d}_{i}^{\perp}\left(\boldsymbol{R}_{\xi}\right)$, and the third as $-\varepsilon_{0}^{-1} \Omega_{i j k}(\xi) \nabla_{k} \nabla_{j} \boldsymbol{d}_{i}{ }^{\perp}\left(\boldsymbol{R}_{\xi}\right)$. Transitions designated as octupolar are comparatively rare, since in any system of less than spherical symmetry most transitions allowed by electric octupole coupling will also be allowed, more strongly, by a lower order multipole. Nonetheless, examples can be found not only in lanthanide ions for example ${ }^{53}$ but also, with surprisingly prominent effect, in argon. ${ }^{54}$ For generality, it is expedient to write the multipole of order $2^{m}$ as a tensor $\boldsymbol{E}_{\xi}^{(m)}$, such that the entire electric multipolar series (5) can be written as a sum of terms of a common form;

$$
H_{\mathrm{int}}(\xi) \equiv \sum_{m} H_{\mathrm{int}}^{(m)}(\xi)=-\varepsilon_{0}^{-1} \sum_{m} E_{\xi ; a_{1} \ldots a_{m}}^{(m)} \nabla_{a_{m}} \ldots \nabla_{a_{2}} \boldsymbol{d}_{a_{1}}^{\perp}\left(\boldsymbol{R}_{\xi}\right)
$$

Time-dependent perturbation theory is now applied with (6) as the perturbation operator; the lowest order non-vanishing contribution comes from second order, since the operator must act twice - namely in the donor decay and the acceptor excitation transitions.

We can now address the electrodynamic coupling between a donor decay and acceptor excitation transition. Each can be ascribed an electric transition multipole, $\mathrm{E} m$ for the donor decay and for the acceptor excitation En, (E1 denoting the electric dipole, E2 the quadrupole, etc.). In practice, such designations generally reflect not the only, but simply the lowest allowed order of multipole - and hence the quantitatively most important - as will be discussed in Section 4. Moreover, in the PZW transformation that delivers the multipolar series from a minimal coupling Hamiltonian, ${ }^{40}$ magnetic multipoles $\mathrm{M} n$ emerge from the from the same order of expansion as $\mathrm{E}(n+1)$. Therefore, even if both are allowed in a given order, the magnetic multipole is generally much weaker than its electric counterpart; this is the reason that magnetic contributions are generally irrelevant for the current considerations. Continuing to focus on electric transition 
multipoles, the quantum amplitude for the process, $M_{f i}^{\mathrm{E} m-\mathrm{E} n}$, is given by:

$$
M_{f i}^{\mathrm{E} m-\mathrm{E} n}=\sum_{r} \frac{\left\langle f\left|H_{\mathrm{int}}^{(m)}(A)\right| r\right\rangle\left\langle r\left|H_{\mathrm{int}}^{(n)}(B)\right| i\right\rangle}{\left(E_{i}-E_{r}\right)}
$$

where $i, f$, and $r$ denote initial, final, and intermediate states of the system and $E$ signifies a corresponding system energy. The general result can be expressed as follows; ${ }^{55,56}$

$$
M_{f i}^{\mathrm{E} m-\mathrm{E} n}=E_{A ; a_{1} \ldots a_{m}}^{(m)} V_{a_{1} \ldots a_{m} b_{1} \ldots b_{n}}(k, \boldsymbol{R}) E_{B ; b_{1} \ldots b_{n}}^{(n)}
$$

The coupling tensor that engages Em donor decay with En acceptor excitation is generally given by;

$$
\begin{aligned}
& V_{a_{1} \ldots a_{m} b_{1} \ldots b_{n}}(k, \boldsymbol{R}) \\
& \quad={\frac{\left(-1 \varepsilon^{m+n-1}\right.}{4 \pi \varepsilon_{0}}}^{m} \nabla_{a_{2}} \ldots \nabla_{a_{m}} \nabla_{b_{2}} \ldots \nabla_{b_{n}}\left(-\nabla^{2} \delta_{a_{1} b_{1}}+\nabla_{a_{1}} \nabla_{b_{1}}\right) \frac{e^{ \pm i k R}}{R},
\end{aligned}
$$

where $k=\Delta E / \hbar c, \Delta E$ is the transferred energy and $\boldsymbol{R}$ is the displacement vector defined by $\boldsymbol{R}_{\mathrm{B}}-\boldsymbol{R}_{\mathrm{A}}$. Immediate notice should be drawn to the non-vanishing character of the result for $m \neq n$, an important possibility that has been overlooked in some literature. In the most common E1-E1 case, (where both the donor and acceptor transitions are electric dipole allowed), implementation of the necessary tensor calculus leads to a result that is concisely expressible as:

$$
M_{f i}^{E 1-E 1}=\mu_{A i} V_{i j}(k, \mathbf{R}) \mu_{B j},
$$

where each $\boldsymbol{\mu}$ is to be understood as a transition dipole, and the E1-E1 coupling tensor is defined by;

$$
\begin{aligned}
V_{i j}(k, \mathbf{R})= & \frac{e^{\mathrm{i} k R}}{4 \pi \varepsilon_{0} R^{3}}\left\{\left(\delta_{i j}-3 \hat{R}_{i} \hat{R}_{j}\right)-(\mathrm{i} k R)\left(\delta_{i j}-3 \hat{R}_{i} \hat{R}_{j}\right)\right. \\
& \left.-(k R)^{2}\left(\delta_{i j}-\hat{R}_{i} \hat{R}_{j}\right)\right\} .
\end{aligned}
$$

Details are given in the original literature, and in a more accessible form by Andrews and Bradshaw. ${ }^{57}$ With a view to the subsequent analysis, notable features of the particular result (11) are the $R^{-3}$ dependence on the donor-acceptor separation distance in the short-range or near-zone $(k R \ll 1)$ and its convergence to $R^{-1}$ in the long-range (the wave-zone, $k R \gg 1$ ); as follows from (1). This is the origin of the $R^{-6}$ and $R^{-2}$ short- and long-range limits of the transfer rate exhibited in Fig. 1, reflecting the quadratic dependence of rate on quantum amplitude.

The above result, and those for the next two higher ranks, can be recast more concisely in terms of spherical Bessel functions whose explicit forms are as given in Appendix 1: ${ }^{55}$

$$
\begin{aligned}
V_{i j}(k, \boldsymbol{R})= & -\frac{i k^{3}}{4 \pi \varepsilon_{0}}\left[\delta_{i j} h_{0}^{(1)}(k R)-(k R)^{-1} \delta_{i j} h_{1}^{(1)}(k R)\right. \\
& \left.+\hat{R}_{i} \hat{R}_{j} h_{2}^{(1)}(k R)\right]
\end{aligned}
$$

$$
\begin{aligned}
& V_{i j k}(k, \mathbf{R})=\frac{i k^{4}}{4 \pi \varepsilon_{0}}\left[\delta_{i j} \hat{R}_{k} h_{1}^{(1)}(k R)\right. \\
&-(k R)^{-1}\left(\delta_{i j} \hat{R}_{k}+\delta_{i k} \hat{R}_{j}+\delta_{j k} \hat{R}_{i}\right) \times h_{2}^{(1)}(k R) \\
&\left.+\hat{R}_{i} \hat{R}_{j} \hat{R}_{k} h_{3}^{(1)}(k R)\right] \\
& V_{i j k l}(k, \mathbf{R})= \frac{i k^{5}}{4 \pi \varepsilon_{0}}\left[\delta_{i j} \delta_{k l} h_{1}^{(1)}(k R)-(k R)^{-2}\right. \\
& \times\left(\delta_{i j} \delta_{k l}+\delta_{i k} \delta_{j l}+\delta_{i l} \delta_{j k}\right) h_{2}^{(1)}(k R) \\
&-\delta_{i j} \hat{R}_{k} \hat{R}_{l} h_{2}^{(1)}(k R)+(k R)^{-1} \\
& \times\left(\delta_{i j} \hat{R}_{k} \hat{R}_{l}+\delta_{i k} \hat{R}_{j} \hat{R}_{l}+\delta_{i l} \hat{R}_{j} \hat{R}_{k}\right. \\
&\left.+\delta_{k l} \hat{R}_{i} \hat{R}_{j}+\delta_{j l} \hat{R}_{i} \hat{R}_{k}+\delta_{j k} \hat{R}_{i} \hat{R}_{l}\right) \\
&\left.\times h_{3}^{(1)}(k R)-\hat{R}_{i} \hat{R}_{j} \hat{R}_{k} \hat{R}_{l} h_{4}^{(1)}(k R)\right]
\end{aligned}
$$

It is noteworthy that the function $h_{j}^{(1)}(k R)$ has terms running from $R^{-1}$ to $R^{-(j+1)}$. Hence the result delivered by (9) has a short-range asymptote running as $R^{-(m+n+1)}$, whereas the wave-zone limit is invariably $R^{-1}$; the latter serves to ensure operation of the inverse square law in radiative energy transfer, irrespective of the multipoles involved in emission and detection.

\section{Irreducible tensor formulation}

The isotropy of atomic systems, which engages irreducible representations (irreps) of the full rotation group $R_{3}$ in simple, one-to-one relationships between angular momentum and multipolar form, supports the familiar development of multipole expansions in a spherical tensor basis. ${ }^{58}$ However, for systems of less than spherical symmetry, such as molecules or atoms in lattice environments that produce crystal field splitting, it is considerably more expedient to tackle angular momentum issues using the tools of irreducible Cartesian tensor analysis. The relationships between the components of tensors in the two representations are well established. ${ }^{59}$ The methods to be described below directly relate to the Cartesian expressions given above, allowing a physically transparent and amenable correlation to be established between angular momentum and multipolar character.

In isolated atoms, states of integer angular momentum transform under the symmetry operations of the full threedimensional rotation group $R_{3}$ or the rotation-inversion group $\mathrm{O}(3)$ in accordance with irreducible representations labelled $S, P, D, \ldots$ for $j=0,1,2, \ldots$ etc. of degeneracy or weight $(2 j+1)$. For an electronic transition to be supported by coupling of a given multipolar form, the product of representations for the initial and final states must be spanned by components of the multipole tensor. In the common case of transitions originating from, or terminating in, a totally symmetric ground state, this reduces to a requirement for one or more irreducible components to have the same transformation properties as the excited state. For example ${ }^{1} S_{0} \leftarrow{ }^{1} D_{2}$ decay in $\mathrm{O}^{2+}$ with $\Delta j=2$ corresponds to an electric quadrupole allowed transition. ${ }^{60}$ 
For molecular systems, necessarily of lower symmetry than atoms, such a correspondence is no less rigorous - but the decay and excitation transition moments typically have components that span more than one irreducible representation of the molecular point group. The general rule for an allowed transition is that the product of the irreducible representations for the initial and final states (for the acceptor the ground and excited states, vice versa for the donor) spans one or more of the representations with which the transition moment components transform, under symmetry operations that reflect the structure of the chromophore and its local electronic environment. Here, the sought transformation properties of the transition multipoles under the operations of the appropriate point group are determined by mapping irreducible representations of the full three-dimensional rotation-inversion group $\mathrm{O}(3)$ onto the corresponding representations of point groups with lower symmetry, usually by reference to correlation tables. ${ }^{61}$

Since the machinery of irreducible Cartesian tensors is to be deployed with regard to not only transition multipoles, but also the electromagnetic fields involved in energy transfer, general features can first be developed with reference to an arbitrary tensor $\boldsymbol{T}_{(n)}$ for which the number of independent Cartesian components is $3^{n}$. The reduction of any such Cartesian tensor results in a sum of irreducible tensors, each characterised by a weight $j \leq n$ and having $(2 j+1)$ independent components; ${ }^{62-64}$ some weights may be represented more than once and are therefore distinguished by a seniority index, $q$;

$$
\boldsymbol{T}_{(n)}=\sum_{j=0}^{n} \sum_{q=1}^{N_{n}^{(j)}} \boldsymbol{T}_{(n)}^{(j ; q)}
$$

The multiplicity of weight $j$ in the reduction scheme, $N_{n}^{(j)}$, is given by the following explicit formula; ${ }^{65}$

$$
N_{n}^{(j)}=\sum_{k}(-1)^{k} \frac{(2 n-3 k-j-2) ! n(n-1)}{(n-3-j) !(n-k) ! k !} .
$$

Here the sum over $k$ is delimited by the condition $0 \leq k \leq\left\lfloor\frac{1}{3}(n-j)\right\rfloor$, the truncated bracket signifying the floor function (integer part). The total number of components in the reduction duly sums to $3^{n}$, as established by the identity:

$$
\sum_{j=0}^{n}(2 j+1) N_{n}^{(j)}=3^{n}
$$

Explicit results for the form of irreducible Cartesian tensors up to rank 4 are available, ${ }^{66}$ and the corresponding transformation properties under each irreducible representation of all the common point groups has been tabulated. ${ }^{67}$ In application to the electric quadrupole, a traceless form can be adopted for the quadrupole, consistent with the transverse character of the electric displacement field $\left(\nabla_{i} \boldsymbol{d}_{i}{ }^{\perp}\left(\boldsymbol{R}_{\xi}\right)=0\right)$.

Given any particular form of coupling, each of the three Cartesian tensors in eqn (8) - the donor decay multipole, the electromagnetic coupling tensor and the acceptor excitation multipole - can be reduced into irreducible parts. It is expedient to focus on the most complex of these, the coupling tensor $\boldsymbol{V}(k, \boldsymbol{R})$. As is evident from the specific results shown in eqns (12)-(14), this has the general structure of a power series in $R^{-1}$, and by exploiting this feature the general result can be cast as follows;

$$
\begin{aligned}
V_{a_{1} \ldots a_{m} b_{1} \ldots b_{n}}(k, \boldsymbol{R})= & \left(4 \pi \varepsilon_{0}\right)^{-1} k^{m+n+1} e^{i k R} \\
& \times \sum_{p=1}^{m+n+1}(k R)^{-p} W_{a_{1} \ldots a_{m} b_{1} \ldots b_{n}}^{(p-1)}(\hat{\boldsymbol{R}}) .
\end{aligned}
$$

Written in this form, the angular momentum character of the electromagnetic coupling is distributed between reducible tensors $\boldsymbol{W}^{(p)}(\hat{\boldsymbol{R}})$, the superscript identifying the associated inverse power of $k R$. Each $\boldsymbol{W}^{(p)}(\hat{\boldsymbol{R}})$ can then be resolved into its own irreducible components of weights $j(p)$;

$$
W_{a_{1} \ldots a_{m} b_{1} \ldots b_{n}}^{(p-1)}(\hat{\boldsymbol{R}})=\sum_{j=0}^{m+n} W_{a_{1} \ldots a_{m} b_{1} \ldots b_{n}}^{(p-1 ; j(p))}(\hat{\boldsymbol{R}}) .
$$

It transpires that the only weights $j(p)$ that are supported in the sum on the right of eqn (19) are those in the range $(p-1 \leq j(p) \leq m+n)$; moreover, only alternate values of $j$ deliver non-zero results. All other terms vanish by virtue of tensor contractions being effected between index-symmetric and correspondingly antisymmetric terms. Accordingly, from the results it is possible to identify correlations between non-zero weights $j(p)$ and the corresponding $R^{-p}$ distance dependence, as shown in Table 1.

To understand the results it is helpful to focus on a specific, non-trivial case. By way of illustration, Fig. 3 exhibits the results obtained for fourth rank coupling, exhibiting the strikingly different forms of distance-dependence associated with angular momentum components identified from eqn (19), running from within the near-zone out into the wave-zone. The implications of such patterns of behaviour can now be explored, particularly with a view to their reconciliation with the angular momentum aspects of the donor and acceptor transitions.

\section{Analysis}

As an overarching principle, the rules of angular momentum coupling, $\left|\boldsymbol{j}_{1}-\boldsymbol{j}_{2}\right| \leq \boldsymbol{j}_{1} \oplus \boldsymbol{j}_{2} \leq \boldsymbol{j}_{1}+\boldsymbol{j}_{2}$, apply to the engagement

\begin{tabular}{|c|c|c|c|c|c|c|c|c|c|c|c|c|}
\hline \multirow{2}{*}{$\frac{\text { Coupling }}{\text { Terms in } R^{-p}}$} & \multicolumn{3}{|c|}{ E1-E1 } & \multicolumn{4}{|c|}{ E2-E1, E1-E2 } & \multicolumn{5}{|c|}{$\mathrm{E} 3-\mathrm{E} 1, \mathrm{E} 2-\mathrm{E} 2, \mathrm{E} 1-\mathrm{E} 3$} \\
\hline & $R^{-1}$ & $R^{-2}$ & $R^{-3}$ & $R^{-1}$ & $R^{-2}$ & $R^{-3}$ & $R^{-4}$ & $R^{-1}$ & $R^{-2}$ & $R^{-3}$ & $R^{-4}$ & $\overline{R^{-5}}$ \\
\hline$j(p)$ & 0,2 & 2 & 2 & 1,3 & 1,3 & 3 & 3 & $0,2,4$ & 2,4 & 2,4 & 4 & 4 \\
\hline
\end{tabular}
of each chromophore's transition moment with the electromagnetic coupling tensor $\boldsymbol{V}$. Following eqn (8) and applying this rule pairwise (in any order) yields a result that necessarily

Table 1 Terms in $R^{-p}$ and corresponding weights $j(p)$ in the $V$ coupling tensors for each of the principal (lowest order) forms of electric multipole coupling: E1-E1 $(m+n=2)$; E2-E1 and E1-E2 $(m+n=3)$; E3-E1, E2-E2 and E1-E3 $(m+n=4)$ 


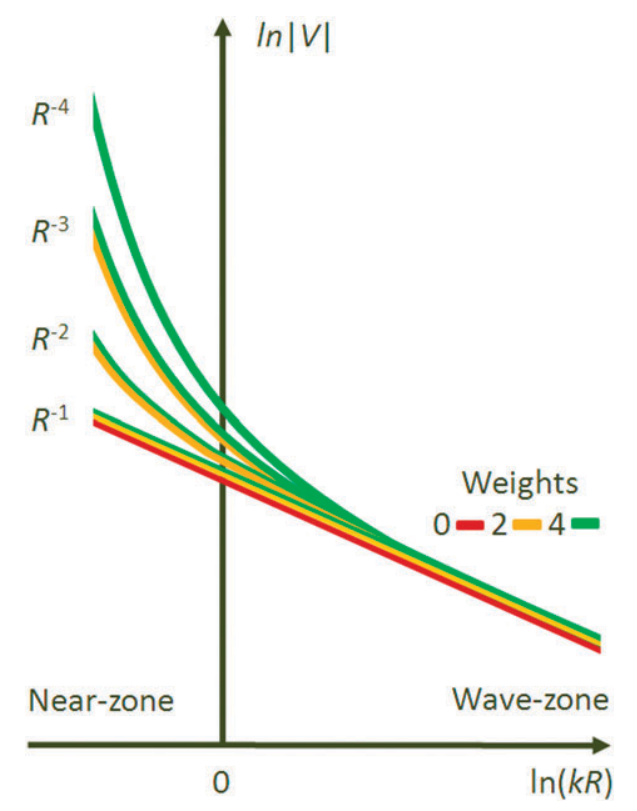

Fig. 3 Indicative $\log -\log$ plot against $k R$ of the fourth rank electromagnetic coupling $V$ connecting electric multipoles $\mathrm{E} m$ and $\mathrm{E} n$, $(m+n=4)$, exhibiting the various contributions having different short-range inverse power dependences on the donor-acceptor distance $R$, also revealing the composition of each angular momentum weight. In the wave-zone asymptote the contributions of all weights run with $R^{-1}$, consistent with an inverse square rate law for radiative energy transfer.

admits a contribution of weight zero; since the result is a scalar (rank zero), any other weight, exceeding the rank, would deliver a vanishing result. Also as a general rule, in the nearzone that is of principal interest for observations of resonance energy transfer, the term with the highest inverse power of $R$ clearly overshadows all other contributions. Thus for E1-E1 coupling, for example, the $R^{-3}$ term which dominates is consistent with a coupling of one unit of angular momentum from the transition dipole at each chromophore (i.e. the donor $A$ and the acceptor $B$ ) with two units, $j(p)=2$, in the $\boldsymbol{V}$ tensor.

It is misleading to regard this first result as indicating an invariably faithful conveyance of the unit angular momentum from $A$ to $B$, however. To understand the wider issue of fidelity, one has to entertain the possibility that other multipolar forms of coupling might also be involved in coupling the same donor and acceptor transitions - which can only be resolved by considering the detailed selection rules that operate for each transition, as determined by the symmetry point group that applies in each location. For example if the donor decay is not only electric dipole but also electric quadrupole allowed, then the finite E2-E1 coupling admits additional $R^{-4}$ contributions to the quantum amplitude for energy transfer, and the rules of angular momentum addition are still fully satisfied. Moreover, other cases can be found where the donor decay is only E2 allowed and the acceptor transition only E1 allowed. To better understand the principles that operate here, it is useful to rehearse the possible combinations of weights that can be simultaneously allowed. The following principles are general and, although illustrative examples are given, a definitive listing of all the possibilities
Table 2 Five-fold categorization of transition symmetries according to their electric multipole character, up to and including the octupole, illustrated by common point groups. In the column on the left, the first entry is the designated character, signifying the lowest allowed order (hence the strongest contribution to the transition); subsequent terms in brackets signify other multipoles that are also allowed, e.g. E2 (E3) denotes a transition symmetry that spans components of both E2 and E3. The absence of a bracketed term denotes that no higher orders of electric multipole (below hexadecapole) are allowed

\begin{tabular}{lllll}
\hline Allowed multipoles & $C_{2 v}$ & $D_{4 h}$ & $T_{d}$ & $C_{\infty v}$ \\
\hline E1 (E2,E3) & $A_{1}, B_{1}, B_{2}$ & & $T_{2}$ & $\Sigma^{+}, \Pi$ \\
E1 (E3) & & $A_{2 u}, E_{u}$ & & \\
E2 & & $A_{1 g}, B_{1 g}, B_{2 g}, E_{g}$ & $E$ & \\
E2 (E3) & $A_{2}$ & $B_{1 u}, B_{2 u}$ & $A, T_{1}$ & $\Phi$ \\
E3 & &
\end{tabular}

that arise for each of the common symmetry point groups is provided in ref. 67, and in Appendix A6.2 of ref. 66. Table 2 exemplifies the results for the common point groups $C_{2 v}, D_{4 h}, T_{d}$ and $C_{\infty v}$.

\section{Electric dipole-allowed transitions}

Electric dipole transitions have transition moments whose Cartesian components provide a basis for weight 1 representations. For any particular transition to admit an electric dipole character almost invariably signals that weight 3 (hence electric octupole) character is also allowed. The sole exceptions amongst all the familiar point groups are cases of icosahedral ( $I$ or $I_{h}$ ) symmetry-reflecting how closely one has to approach full spherical symmetry before the different weights are entirely separable. Nonetheless, for an E1-allowed transition, additional contributions (whether of E2, E3 or higher multipolar form) are considerably weaker in effect, and their presence makes no difference to the primary designation.

\section{Electric quadrupole-allowed transitions}

Transitions that are designated as electric quadrupolar have transformation properties that necessarily admit weight 2; according to the local symmetry, weight 1 may additionally be permissible under the same, corresponding irreducible representation. The two possibilities for quadrupole-allowed transitions are therefore that they are either allowed by weight 2 alone (and though of little interest, conceivably by higher orders), or by both weights 2 and 1 . In the latter case a further distinction can be drawn between chiral and achiral centres. In an achiral centre, electric dipoles and their weaker magnetic counterparts transform differently, and in consequence some transitions that are E1-forbidden can be both electric quadrupole and magnetic dipole allowed (e.g. $A_{2}$ transitions in molecules of $C_{2 v}$ symmetry). However, for reasons that were explained in Section 2, in such cases magnetic dipoles and electric quadrupoles can give broadly similar contributions to the quantum amplitude; as such, these transitions are still meaningfully designated as having electric quadrupolar character. So, the remaining cases of interest are either those transitions that are both electric dipole and electric quadrupole allowed - in which case they are designated by the lower order and more prominent dipole, and the electric 
quadrupolar component is a minor correction - or they are allowed by weight 2 at lowest order (as for example in transitions of $B_{1 g}$ or $B_{2 g}$ symmetry in $D_{4 h}$ species).

\section{Electric octupole-allowed transitions}

Here weight 3 is necessarily allowed; the possible combinations are (a) 3 alone; (b) 3 and 2; (c) 3 and 1; (d) 3, 2 and 1. The last two of these cases relate to transitions that are necessarily electric dipole allowed (the parity is the same as that which admits the octupole, so there is no need to consider even parity magnetic dipoles). These are transitions for which the presence of other contributions only signifies small corrections. Once more in case $(b)$ it is possible to identify a different behaviour in chiral and achiral chromophores; in achiral species, electric and magnetic quadrupoles transform differently and some transitions may be both electric octupole and magnetic quadrupole allowed (e.g. a $B_{1 u}$ or $B_{2 u}$ transition in $D_{4 h}$ symmetry); again, the magnitudes of the two forms of coupling are broadly similar and the electric octupolar descriptor is valid. Thus it is only cases $(a)$ and $(b)$ that demand attention, and the latter is none other than an electric quadrupole transition with an octupolar correction. The key electric octupole transitions of interest are therefore those of type (a) - those allowed by weight 3 alone.

The results for the case of $m+n=4$ coupling, as exhibited in Fig. 3, are particularly illuminating. The same fourth-rank form of electromagnetic coupling tensor mediates E3-E1 and E1-E3, and also E2-E2 energy transfer. Revisiting the corresponding results in Table 1, it might be considered that the weight 0 term, which delivers the least significant component of the quantum amplitude in the usual distance regime for energy transfer, should only satisfy the rule for the addition of angular momenta in the E2-E2 case. However, the $j(p)=0$ contribution does not vanish in either case of E3 transfer, because the electric octupole tensor is not fully pairwise traceless; a weight 1 part of the octupole persists ${ }^{68}$ and as a result, the weight 0 part of the coupling tensor does not compromise the rules of angular momentum coupling.

\section{Conclusion}

In the majority of materials in which resonance energy transfer is observed, the participating chromophores have less than spherical symmetry, and for any such species the electronic state transitions are commonly mediated by more than one form of electric multipole. In the light of the present analysis, it is clear that finite quantum amplitudes routinely arise for the transfer of energy between decay and excitation transition multipoles of different order, even in the exceptional cases where a specific multipole is uniquely allowed, to a given order. It is not generally possible to secure a one-to-one correlation between the transition multipoles involved in source decay and detector excitation; any inference of a faithful conveyance of orbital angular momentum, between energy donor and acceptor, is critically undermined. Nonetheless, with the electromagnetic coupling properly understood, it is evident that the rules of angular momentum addition are indeed faithfully adhered to. The overall result reflects the fact that angular momentum is locally conserved in both the source emission and detector excitation quantum transitions. Since these transitions are individually no different in character from those that can occur in individual optical emission and absorption processes, the conclusion is consistent with the results of a recent analysis of radiative transitions, based on vector spherical harmonics. ${ }^{69}$ In the context of electronic energy transfer, however, it is highly significant that that local isotropy does not extend to the combined source-detector system, and there are indeed differences in form between spherical radiation modes centred on spatially translated donor and acceptor locations. ${ }^{70}$ This is one reason why a Cartesian tensor basis is more conducive for the analysis. As has been shown, the key result is that angular momentum associated with donor decay cannot be assumed to identify with that involved in a corresponding acceptor excitation.

Further, ongoing work in this area is assessing the implications for the long-range asymptotes of the electromagnetic coupling. ${ }^{71}$ In connection with this distance regime, which relates to radiative coupling, a consideration of the fidelity of angular momentum transfer leads into a new raft of issues concerning the character of the photons that propagate between any source and detector of different multipolar operation. In this connection, for example, it has already proven possible to correct a surprisingly prevalent notion that the detection of radiative emission of a particular multipolar order should require a detector having an excitation of matching multipolarity. As has recently been shown, ${ }^{71}$ the resolution of this and other closely related issues may relate at a yet more fundamental level to angular momentum uncertainty. It appears that the correct understanding establishes a fundamental limitation on the propagation of multipolar character, and a new constraint on angular momentum-based information delivery in long-range quantum communication.

\section{Appendix 1: Spherical Bessel functions}

The general form of the spherical Bessel functions has the following series representation;

$$
h_{j}^{(1)}(z)=i^{-(j+1)} \frac{e^{i z}}{z} \sum_{p=0}^{j}\left(\frac{i}{2 z}\right)^{p} \frac{(j+p) !}{p !(j-p) !}
$$

giving the following explicit results required by eqn (12)-(14);

$$
\begin{aligned}
& h_{0}^{(1)}(k R)=-i e^{i k R}\left(\frac{1}{k R}\right) \\
& h_{1}^{(1)}(k R)=-e^{i k R}\left(\frac{1}{k R}+\frac{i}{k^{2} R^{2}}\right) \\
& h_{2}^{(1)}(k R)=e^{i k R}\left(\frac{i}{k R}-\frac{3}{k^{2} R^{2}}-\frac{3 i}{k^{3} R^{3}}\right) \\
& h_{3}^{(1)}(k R)=e^{i k R}\left(\frac{1}{k R}+\frac{2 i}{k^{2} R^{2}}-\frac{15}{k^{3} R^{3}}-\frac{15 i}{k^{4} R^{4}}\right) \\
& h_{4}^{(1)}(k R)=e^{i k R}\left(\frac{-i}{k R}+\frac{10}{k^{2} R^{2}}+\frac{45 i}{k^{3} R^{3}}-\frac{105}{k^{4} R^{4}}-\frac{105 i}{k^{5} R^{5}}\right)
\end{aligned}
$$




\section{Acknowledgements}

I thank David Bradshaw and Luciana Dávila Romero for helpful comments and a careful reading of the manuscript.

\section{References}

1 J. S. Avery, Resonance energy transfer and spontaneous photon emission, Proc. Phys. Soc., 1966, 88, 1-8.

2 L. Gomberoff and E. A. Power, The resonance transfer of excitation, Proc. Phys. Soc., 1966, 88, 281-284.

3 B. W. van der Meer, J. Coker III and S.-Y. S. Chen, Resonance Energy Transfer: Theory and Data, VCH, New York, 1982.

4 D. L. Andrews and A. A. Demidov, Resonance Energy Transfer, Wiley, Chichester, 1999.

5 G. D. Scholes, Long-range resonance energy transfer in molecular systems, Annu. Rev. Phys. Chem., 2003, 54, 57-87.

6 D. L. Andrews, Mechanistic principles and applications of resonance energy transfer, Can. J. Chem., 2008, 86, 855-870.

7 G. W. Robinson and R. P. Frosch, Electronic excitation transfer and relaxation, J. Chem. Phys., 1963, 38, 1187-1203.

8 C.-P. Hsu, G. R. Fleming, M. Head-Gordon and T. HeadGordon, Excitation energy transfer in condensed media, J. Chem. Phys., 2001, 114, 3065-3072.

9 V. May, Beyond the Förster theory of excitation energy transfer: importance of higher-order processes in supramolecular antenna systems, Dalton Trans., 2009, 10086-101005.

10 W. C. Martin and W. L. Wiese, in Atomic, Molecular, and Optical Physics Handbook, ed. G. W. F. Drake, AIP, Woodbury NY, 1996, ch. 10 , pp. $135-153$.

11 D. L. Andrews and B. S. Sherborne, Resonant excitation transfer: A quantum electrodynamical study, J. Chem. Phys., 1987, 86, 4011-4017.

12 D. L. Andrews, A unified theory of radiative and radiationless molecular energy transfer, Chem. Phys., 1989, 135, 195-201.

13 G. Juzeliūnas and D. L. Andrews, Quantum electrodynamics of resonant energy transfer in condensed matter, Phys. Rev. B: Condens. Matter, 1994, 49, 8751-8763.

14 E. A. Power and T. Thirunamachandran, Analysis of the causal behaviour in energy transfer between atoms, Phys. Rev. A: At., Mol., Opt. Phys., 1997, 56, 3395-3408.

15 G. Juzeliūnas and D. L. Andrews, Quantum electrodynamics of resonance energy transfer, Adv. Chem. Phys., 2000, 112, 357-410.

16 G. J. Daniels, R. D. Jenkins, D. S. Bradshaw and D. L. Andrews, Resonance energy transfer: The unified theory revisited, J. Chem. Phys., 2003, 119, 2264-2274.

17 A. Salam, Resonant transfer of excitation between two molecules using Maxwell fields, J. Chem. Phys., 2005, 122, 044113.

18 L. Allen, M. W. Beijersbergen, R. J. C. Spreeuw and J. P. Woerdman, Orbital angular momentum of light and the transformation of Laguerre-Gaussian laser modes, Phys. Rev. A: At., Mol., Opt. Phys., 1992, 45, 8185-8189.

19 L. Mandel and E. Wolf, Optical Coherence and Quantum Optics, University Press, Cambridge, 1995, Sect. 10.6.2.

20 L. Allen, M. J. Padgett and M. Babiker, The optical angular momentum of light, Prog. Opt., 1999, 39, 291-372.

21 L. Allen, S. M. Barnett and M. J. Padgett, Optical Angular Momentum, Institute of Physics, Bristol, 2003.

22 D. L. Andrews, Structured Light and its Applications: An Introduction to Phase-Structured Beams and Nanoscale Optical Forces, Academic, Burlington MA, 2008.

23 S. M. Barnett, Optical angular-momentum flux, J. Opt. B: Quantum Semiclassical Opt., 2002, 4, S7-S16.

24 J. Leach, M. J. Padgett, S. M. Barnett, S. Franke-Arnold and J. Courtial, Measuring the orbital angular momentum of a single photon, Phys. Rev. Lett., 2002, 88, 257901.

25 J. Leach, J. Courtial, K. Skeldon, S. M. Barnett, S. Franke-Arnold and M. J. Padgett, Interferometric methods to measure orbital and spin, or the total angular momentum of a single photon, Phys. Rev. Lett., 2004, 92, 013601.

26 C. R. Kagan, C. B. Murray, M. Nirmal and M. G. Bawendi, Electronic energy transfer in CdSe quantum dot solids, Phys. Rev. Lett., 1996, 76, 1517-1520.
27 S. A. Crooker, J. A. Hollingsworth, S. Tretiak and V. I. Klimov, Spectrally resolved dynamics of energy transfer in quantum-dot assemblies: Towards engineered energy flows in artificial materials, Phys. Rev. Lett., 2002, 89, 186802.

28 B. W. Lovett, J. H. Reina A. Nazir and G. A. D. Briggs, Optical schemes for quantum computation in quantum dot molecules, Phys. Rev. B: Condens. Matter Mater. Phys., 2003, 68, 205319.

29 H. Kamada and H. Gotoh, Quantum computation with quantum dot excitons, Semicond. Sci. Technol., 2004, 19, S392-S396.

30 S. Sangu, K. Kobayashi, A. Shojiguchi and M. Ohtsu, Logic and functional operations using a near-field optically coupled quantum-dot system, Phys. Rev. B: Condens. Matter Mater. Phys., 2004, 69, 115334.

31 A. R. Clapp, I. L. Medintz, H. T. Uyeda, B. R. Fisher, E. R. Goldman, M. G. Bawendi and H. Mattoussi, Quantum dot-based multiplexed fluorescence resonance energy transfer, J. Am. Chem. Soc., 2005, 127, 18212-18221.

32 J. M. Villas-Boas, S. E. Ulloa and A. O. Govorov, Photocurrent and spin manipulation in quantum dots, Phys. E., 2006, 34, 333-335.

33 M. Lunz, A. L. Bradley, W.-Y. Chen and Y. K. Gun'ko, Förster resonant energy transfer in quantum dot layers, Superlattices Microstruct., 2010, 47, 98-102.

34 G. D. Scholes, D. L. Andrews, V. M. Huxter, J. Kim and C. Y. Wong, Transmission of quantum dot exciton spin states via resonance energy transfer, Proc. SPIE, 2005, 5929, 86-93.

35 G. D. Scholes and D. L. Andrews, Resonance energy transfer and quantum dots, Phys. Rev. B: Condens. Matter Mater. Phys., 2005, 72, 125331.

36 R. Baer and E. Rabani, Theory of resonance energy transfer involving nanocrystals: The role of higher multipoles, J. Chem. Phys., 2008, 128, 184710.

37 D. L. Andrews and J. M. Leeder, Resonance energy transfer: When a dipole fails, J. Chem. Phys., 2009, 130, 184504.

38 E. A. Power, Introductory Quantum Electrodynamics, Longmans, London, 1964.

39 W. P. Healy, Non-Relativistic Quantum Electrodynamics, Academic, London, 1982.

40 C. Cohen-Tannoudji, J. Dupont-Roc and G. Grynberg, Photons and Atoms. Introduction to Quantum Electrodynamics, WileyInterscience, New York, 1989, pp. 45-60.

41 D. P. Craig and T. Thirunamachandran, Molecular Quantum Electrodynamics, Dover, New York, 1998.

42 A. Salam, Molecular Quantum Electrodynamics: Long-Range Intermolecular Interactions, Wiley, Hoboken NJ, 2010.

43 R. G. Woolley, Charged particles, gauge invariance and molecular electrodynamics, Int. J. Quantum Chem., 1999, 74, 531-545.

44 J. K. Jenkins and S. Mukamel, Quantum electrodynamics of molecular nanostructures, J. Chem. Phys., 1993, 98, 7046-7058.

45 A. E. Cohen and S. Mukamel, A mechanical force accompanies fluorescence resonance energy transfer (FRET), J. Phys. Chem. A, 2003, 107, 3633-3638.

46 D. L. Andrews, D. S. Bradshaw, J. Leeder and J. Rodríguez, Dynamics of the dispersion interaction in an energy transfer system, Phys. Chem. Chem. Phys., 2008, 10, 5250-5255.

47 D. S. Bradshaw and D. L. Andrews, Optically controlled resonance energy transfer: Mechanism and configuration for all-optical switching, J. Chem. Phys., 2008, 128, 144506.

48 D. S. Bradshaw and D. L. Andrews, All-optical switching between quantum dot nanoarrays, Superlattices Microstruct., 2010, 47, 308-313.

49 R. G. Woolley, Reformulation of molecular quantum electrodynamics, J. Phys. B: At. Mol. Phys., 1974, 7, 488-499.

50 E. A. Power and T. Thirunamachandran, Nature of Hamiltonian for interaction of radiation with atoms and molecules, Am. J. Phys., 1978, 46, 370-378.

51 V. Chernyak and S. Mukamel, Gauge invariant formulation of molecular electrodynamics and the multipolar Hamiltonian, Chem. Phys., 1995, 198, 133-143.

52 M. Babiker, E. A. Power and T. Thirunamachandran, On a generalization of the Power-Zienau-Woolley transformation in quantum electrodynamics and atomic field equations, Proc. $R$. Soc. London, Ser. A, 1974, 338, 235-249. 
53 M. Roberts, P. Taylor, G. P. Barwood, P. Gill, H. A. Klein and W. R. C. Rowley, Observation of an electric octupole transition in a single ion, Phys. Rev. Lett., 1997, 78, 1876-1879.

54 P. Lundin, J. Gurell, L.-O. Norlin, P. Royen, S. Mannervik, P. Palmeri, P. Quinet, V. Fivet and E. Biemont, Inclusion of electric octupole contributions explains the fast radiative decays of two metastable states in $\mathrm{Ar}^{+}$, Phys. Rev. Lett., 2007, 99, 213001.

55 G. D. Scholes and D. L. Andrews, Damping and higher multipole effects in the quantum electrodynamical model for electronic energy transfer in the condensed phase, J. Chem. Phys., 1997, 107, 5374-5384.

56 A. Salam, A general formula for the rate of resonant transfer of energy between two electric multipole moments of arbitrary order using molecular quantum electrodynamics, J. Chem. Phys., 2005, 122, 044112.

57 D. L. Andrews and D. S. Bradshaw, Virtual photons, dipole fields and energy transfer: A quantum electrodynamical approach, Eur. J. Phys., 2004, 25, 845-858.

58 J.-M. Normand, A Lie Group: Rotations in Quantum Mechanics, North-Holland, Amsterdam, 1980, Appendix H.

59 J.-M. Normand and J. Raynal, Relations between Cartesian and spherical components of irreducible Cartesian tensors, J. Phys. A: Math. Gen., 1982, 15, 1437-1461.

60 R. D. Cowan, The Theory of Atomic Structure and Spectra, University of California Press, CA, 1981, p. 451.

61 R. L. Carter, Molecular Symmetry and Group Theory, Wiley, Chichester, 1998, ch. 7, and Appendix B.
62 J. A. R. Coope, R. F. Snider and F. R. McCourt, Irreducible Cartesian tensors, J. Chem. Phys., 1965, 43, 2269-2275.

63 J. Jerphagnon, D. Chemla and R. Bonneville, The description of the physical properties of condensed matter using irreducible tensors, Adv. Phys., 1978, 27, 609-650.

64 T. Damour and B. R. Iyer, Multipole analysis for electromagnetism and linearized gravity with irreducible Cartesian tensors, Phys. Rev. D: Part., Fields, Gravitation, Cosmol., 1991, 43, 3259-3272.

65 V. V. Mihailov, Addition of arbitrary number of identical angular momenta, J. Phys. A: Math. Gen., 1977, 10, 147-153.

66 D. L. Andrews and P. Allcock, Optical Harmonics in Molecular Systems, Wiley-VCH, Weinheim, 2002, Appendix 4.

67 D. L. Andrews, Symmetry characterisation in molecular multiphoton spectroscopy, Spectrochim. Acta, 1990, 46A, 871-885.

68 A. Salam and T. Thirunamachandran, A new generalization of the Casimir-Polder potential to higher electric multipole polarizabilities, J. Chem. Phys., 1996, 104, 5094-5099.

69 R. Grinter, Photon angular momentum: selection rules and multipolar transition moments, J. Phys. B: At., Mol. Opt. Phys., 2008, 41, 095001.

70 G. Molina-Terriza, J. P. Torres and L. Torner, Management of the angular momentum of light: Preparation of photons in multidimensional vector states of angular momentum, Phys. Rev. Lett., 2001, 88, 013601.

71 D. L. Andrews, Optical angular momentum: Multipole transitions and photonics, Phys. Rev. A: At., Mol., Opt. Phys., 2010, 81, 033825 . 